\title{
Electrically Controlled Rotor Blade Vortex Interaction Airloads and Noise Analysis Using Viscous Vortex Particle Method
}

\author{
Taoyong Su, Yang Lu $(D$, Jinchao Ma, and Shujun Guan \\ National Key Laboratory of Rotorcraft Aeromechanics, College of Aerospace Engineering, \\ Nanjing University of Aeronautics and Astronautics, Nanjing 210016, China \\ Correspondence should be addressed to Yang Lu; njluyang@163.com
}

Received 22 August 2019; Accepted 21 October 2019; Published 6 November 2019

Academic Editor: Angelo Marcelo Tusset

Copyright () 2019 Taoyong Su et al. This is an open access article distributed under the Creative Commons Attribution License, which permits unrestricted use, distribution, and reproduction in any medium, provided the original work is properly cited.

\begin{abstract}
An electrically controlled rotor (ECR), also called a swashplateless rotor, replaces a swashplate with a trailing-edge flap system to implement primary rotor control. To investigate the aerodynamic characteristics of an ECR in blade-vortex interaction (BVI) condition, an analysis model based on the viscous vortex particle method, ECR blade pitch equation, and the Weissinger-L lifting surface model is established. In this model, the ECR wake flow field vorticity is discretized as multiple vortex particles, and the vorticity-velocity form of the Navier-Stokes equation is solved to simulate the transport diffusion of the vorticity. The flap motioninducing blade-pitch movement is obtained by solving the ECR blade-pitch movement equation via the Runge-Kutta fourthorder method. On the basis, BVI noise radiation of an ECR is evaluated using the Ffowcs Williams and Hawkings (FW-H) equation. Based on the present prediction model, the aerodynamic and acoustic characteristics of a sample ECR in BVI condition are analyzed. The results show that since the BVI event of the ECR on the advancing side is mainly caused by the interaction between the flap tip vortex and the blade, the blade spanwise range of ECR BVI occurrence on the advancing side is smaller than that of the conventional rotor. In addition, the magnitude of the maximum sound pressure level on the advancing side as well as on the retreating side of the ECR is also different from that of the conventional rotor, which is consistent with the difference in the airloads between the ECR and conventional rotor. Furthermore, a study was performed to examine the effect of the pre-index angle on the BVI-induced airloads and noise. The amplitude of the impulsive airloads of the ECR on the advancing side is increased with the increase in pre-index angle, while the amplitude of the impulsive airloads of the ECR on the retreating side is decreased. Indeed, when the pre-index angle of the sample ECR is 8 degrees, the retreating-side noise radiation lobe is almost disappeared. In addition, the different intensity of wake vorticity is the main reason for the differences of the BVI-induced airloads and noise among the ECR with different pre-index angles.
\end{abstract}

\section{Introduction}

Electrically controlled rotor (ECR), also called as swashplateless rotor, applies blade pitch inputs via trailing-edge flap system instead of traditional swashplate mechanism [1]. As the swashplate is eliminated, the control system of the ECR can be simplified, which can effectively reduce the empty weight and the parasite drag of the helicopter [2]. In addition to primary control, applying harmonic or nonharmonic motions, the trailing-edge flap system could also be used for rotor vibration reduction $[3,4]$, noise alleviation [5-7], and performance enhancement $[8,9]$.
The helicopter is the quietest vertical take-off and landing (VTOL) aircraft, but its noise level can still be high enough to compromise its utility unless specific attention is given to designing for low noise [10]. Rotor noise is one of the main sources of helicopter noise and is usually divided into the deterministic components of thickness noise and loading noise, blade-vortex interaction (BVI) noise, highspeed impulsive (HSI) noise, and broadband noise. Among them, BVI noise is essentially a specific type of loading noise, which has an impulsive nature. When a rotor operates in the descent or maneuvering flight, the strong tip vortex released by a blade will approach or even pass through the rotor disc 
plane and interact with the following blade, resulting in a strong BVI event. This BVI event will lead to impulsive changes in blade airloads, which will not only increase the vibration level of the rotor but also generate BVI noise. Once BVI noise appears, it will extremely annoy people and significantly increase the overall noise level of the helicopter. Therefore, alleviating the BVI noise is always the research focus in the field of helicopter aeroacoustics. With the trailing-edge flap system, ECR can theoretically alleviate BVI noise by using active control technology. However, so far researches on ECR have mainly focused on feasibility analysis [11], aerodynamic modeling $[1,2,12,13]$, design parameter analysis [14], and performance enhancement [15]. There is no relevant research on the BVI airloads and noise characteristics of an ECR. In fact, because of flap motion, the ECR wake flow field is more complex than that of a conventional rotor, which subsequently affects the distributions of the induced inflow and airloads. Improving the basic understanding of BVI phenomenon of ECR is helpful to alleviate the BVI noise by using active bladecontrol techniques.

During the last several decades, considerable efforts have been focused on experiments and numerical prediction of conventional rotor BVI-induced airloads and noise [16-18]. On this foundation, the best-known HART II test campaign was conducted at the DNW low-speed wind tunnel in 2001 by a joint cooperation between DLR, Onera, DNW, US Army AFDD, and NASA Langley, which provide experimental insight into the aeroelastic and noise characteristics of a conventional rotor in BVI condition [19-21]. The effects of the higher harmonic pitch control (HHC) inputs on the rotor vibration and BVI noise were also investigated in the experiment. Based on the experimental database, which includes blade elastic deformation, sectional airloads, wake geometry, tip vortex strength, and acoustic radiation measurements, a series of analytical models for the prediction of rotor BVI-induced airloads and noise have been assessed. Incorporating the free wake model into the nonlinear flexible multibody dynamics analysis code, Park compared the predicted results with the HART II experimental data [22]. Gennaretti predicted the BVI-induced airloads and noise using the panel/full-span free-wake coupled method [23]. Compared with the freewake model, the full-span free-wake model can capture the BVI event more accurately on the advancing and retreating sides. Besides, the full-span free-wake model can also improve the prediction of tip vortices trajectories. However, in Gennaretti's research, the vortex core size still needs to be determined preliminarily. With the development of CFD techniques, many researchers have used CFD method to investigate the aerodynamic and acoustic characteristics of helicopter rotor BVI. References [24, 25] show encouraging results in comparison with experimental data for their prediction of BVI-induced airloads from the HART II test. Furthermore, an assessment of the state of the art of CFD-CSD approach for predicting BVI event is provided in reference [26]. Although CFD method has unique advantages in solving rotor blade airloads, the inherent numerical dissipation of most CFD requires very high grid densities to maintain the fidelity of the wake, particularly if the structure of the wake is to be resolved to a level where the high-frequency character of the BVI-induced airloads can be captured. Therefore, in recent years, researchers have adopted some alternative methods to investigate the BVI phenomenon. Kelly conducted a comprehensive investigation on the application of vorticity transport model (VTM) to the rotor BVI-induced airloads and noise analysis $[27,28]$. The effects of the lifting-line model and the lifting-chord model on the high-frequency sectional airloads as well as the acoustic are also explored. The numerical results show that the VTM is capable of capturing the tip vortices and their roll-up process. Based on the OLS experimental data, Zhao et al. investigated the ability of hybrid method to predict rotor BVI-induced airloads and noise [29]. In addition, the Lattice-Boltzmann method (LBM) was adopted in reference [30] to establish the analytical model. Based on the experimental database from HART II test, the investigation of the rigid-blade model and elastic-blade model effects on the predicted airloads are also performed.

Accurate prediction of the strength and position of the tip vortices is essential to capture the high-frequency oscillating blade airloads induced by BVI. In recent years, viscous vortex particle method (VVPM) has been widely employed in helicopter aerodynamic analysis [31-36]. This method does not rely on empirical parameters and can take the wake viscous effect into account. In addition, since the vorticity-velocity form of incompressible Navier-Stokes equations are solved directly in the Lagrangian frame, the VVPM can avoid the numerical dissipation problem and is an ideal method for accurately predicting the wake vorticity structure in BVI condition. In this paper, based on the VVPM, ECR blade pitch movement equation, and Weissinger-L lifting surface model, a BVI-induced airloads prediction model for ECR is established. BVI noise radiation of the ECR is evaluated using a postprocessor for the blade airloads that implements Farassat's formulation 1A [37] of the Ffowcs Williams and Hawkings (FW-H) equation [38].

This paper consists of the following sections. In Section 2, first, the ECR blade pitch movement equation is provided. Next, the BVI-induced airloads prediction model of ECR established from the VVPM and Weissinger-L lifting surface is elaborated, and the Farassat $1 \mathrm{~A}$ integral formulation for noise radiation is described. As there is no literature regarding the BVI phenomenon of ECR, in Section 3, first, the HART II experimental rotor is used as an example to validate the aerodynamics and acoustics predicted model established in this paper. On this basis, the BVI characteristics of ECR as well as the effect of the pre-index angle of the blade on it is investigated. Finally, conclusions are outlined in Section 4.

\section{ECR BVI-Induced Airloads and Noise- Prediction Model}

2.1. ECR Blade Pitch Movement Equation. ECR achieves blade pitch movement via pitch moment generated by flap 
deflection, and the blade root usually has very low torsional stiffness. Based on the rigid blade model, the pitch movement equation of the ECR is established, and the torsional stiffness of the blade is determined only by the spring at the blade root. Assuming that the gravity center, the aerodynamic center and the elastic center of the blade are located at the $1 / 4$ chord length, the pitch movement of the blade can be expressed as follows:

$$
I_{f} \ddot{\theta}_{f}+I_{f} \Omega^{2}\left(\theta_{\mathrm{pre}}+\theta_{f}\right)+k_{\theta} \theta_{f}=M_{\theta} .
$$

In the equation, $\theta_{f}$ is the flap deflection and tennis racket moment induced blade pitch angle; $I_{f}$ is the blade rotational inertia around the pitch axis; $k_{\theta}$ is the blade root torsion stiffness coefficient; $\Omega$ is the rotor rotating speed; $\theta_{\text {pre }}$ is the blade pre-index angle which is the blade pitch angle when the ECR does not rotate; and $M_{\theta}$ is the completed blade aerodynamics pitching moment, which include the unsteady pitching moment of the blade and the incremental pitching moment caused by trailing edge flap motion. The unsteady pitching moment of the airfoil is calculated by using the Leishman-Beddoes model [39] and the flap deflection-induced airfoil-pitching moment is calculated via the Hariharan-Leishman unsteady-flap aerodynamics model [40]. The blade pitch movement equation is solved via the Runge-Kutta fourth-order method.

2.2. Viscous Vortex Particle Wake Model of ECR. The velocity-vorticity form of incompressible Navier-Stokes equation in the Lagirange frame can be used to represent the rotor-wake vortex flow with a high Reynolds number. In VVPM, the continuous vorticity field is discretized by using vortex particles with certain vorticity. Thus, the governing equation can be expressed in the form of convection-diffusion of $N$ vortex particles:

$$
\begin{aligned}
& \frac{\mathrm{D} x_{i}}{\mathrm{D} t}=\mathbf{u}\left(\mathbf{x}_{i}, t\right), \\
& \frac{\mathrm{D} \boldsymbol{\alpha}_{i}}{\mathrm{D} t}=\boldsymbol{\alpha}_{i} \cdot \nabla \mathbf{u}\left(\mathbf{x}_{i}, t\right)+\nu \nabla^{2} \boldsymbol{\alpha}_{i}+S,
\end{aligned}
$$

where $\boldsymbol{\alpha}_{i}$ and $\mathbf{x}_{i}$ are a vortex particle vorticity vector and a position vector, respectively, $\mathbf{u}$ is local velocity vector, $v$ is the kinematic viscosity, $\mathrm{D} / \mathrm{D} t$ is the substance derivative, and $S$ is the local vorticity source.

In formula (3), the first term on the right is the stretching-effect term, which represents the effect of stretching and rotation on local vorticity, the second term is the viscous diffusion term, which reflects the viscosity induced the vorticity diffusion effect, and the third term is the source term, which indicates that new vortex particles are constantly generated on the blade surface and shed into the rotor wake. The velocity gradient in the stretching term includes the rotor wake vortex particle and rotor blade induced velocity gradient. The vortex particle induced velocity gradient is solved directly:

$$
\boldsymbol{\alpha}_{i} \cdot \nabla \mathbf{u}_{i p}\left(\mathbf{x}_{i}, t\right)=\left[\sum_{j=1}^{N} \frac{1}{\sigma_{i j}^{3}}\left[\widetilde{\boldsymbol{\alpha}}_{j}\right]\left[\nabla\left(K(\rho)\left(\mathbf{x}_{i}-\mathbf{x}_{j}\right)\right)\right]\right] \cdot \boldsymbol{\alpha}_{i},
$$

and the viscous diffusion is determined via a particle strength exchange (PSE) method:

$$
\nu \nabla^{2} \boldsymbol{\alpha}_{i}=\frac{2 v}{\sigma_{i j}^{2}} \sum_{j=1}^{N}\left(V_{i} \boldsymbol{\alpha}_{j}-V_{j} \boldsymbol{\alpha}_{i}\right) \xi_{\sigma_{i j}}\left(\mathbf{x}_{i}-\mathbf{x}_{j}\right),
$$

where $\left[\widetilde{\boldsymbol{\alpha}}_{j}\right]$ is an antisymmetric tensor composed of the vorticity vector of a vortex particle, $\sigma_{i j}$ is the symmetric smoothing parameter, $K(\rho)$ is the Biot-Savart kernel function after the smoothing correction, $V$ is the volume of a vortex particle, and $\xi_{\sigma}\left(\mathbf{x}-\mathbf{x}_{i}\right)$ is the vortex particle vorticity distribution function after a smoothing correction. The detailed expressions of the symmetric smoothing parameter $\sigma_{i j}$, the Biot-Savart kernel function after the smoothing correction $K(\rho)$, and the vortex particle vorticity distribution function after a smoothing correction $\xi_{\sigma}\left(\mathbf{x}-\mathbf{x}_{i}\right)$ are provided in the references $[1,31]$. The expression of the source term will be provided in the next section.

2.3. Weissinger-L ECR Blade Lifting Surface Model. The rotor-wake flow field vorticity convection and diffusion process are simulated via the VVPM, and the specific form of the source term depends on the blade aerodynamic model. In this paper, the Weissinger- $\mathrm{L}$ lifting surface model is employed to solve the variation in the ECR blade bound vortex circulation along the span direction, which in turn acquires the source term $S$ in formula (3).

Figure 1 shows the solution diagram of the ECR blade bound vortex lattice and new vortex particle. The bound circulation of blade segments usually varies both spanwise and azimuthally, which generates the trailed and shed vorticity from the blades. When calculating the blade bound vortex circulation distribution at the current azimuth, the near wake is still discretized via the vortex lattice and its circulation is equal to the bound circulation of the blade segment corresponding to the previous azimuth. The position of the vortex lattice trailing edge is determined by the local flow velocity.

To solve the blade bound vortex circulation at each segment, a control point is deployed at the $3 / 4$ blade chord in each vortex lattice. Based on an impenetrable boundary condition, the relation between the bound vortex circulation and locally induced velocity can be represented as follows:

$$
\mathbf{A} \Gamma_{\mathrm{b}} \mathbf{n}=\mathbf{B n},
$$

where $\mathbf{A}$ is the influence coefficients matrix of the bound vortex lattice on the control point, $\Gamma_{\mathrm{b}}$ is the bound vortex circulation matrix, $\mathbf{B}$ is the matrix of the velocity induced by the near wake vortex lattice, wake vortex particle, and blade movement on the blade control point, and $\mathbf{n}$ is the outward unit normal vector of the blade surface at the control point. 


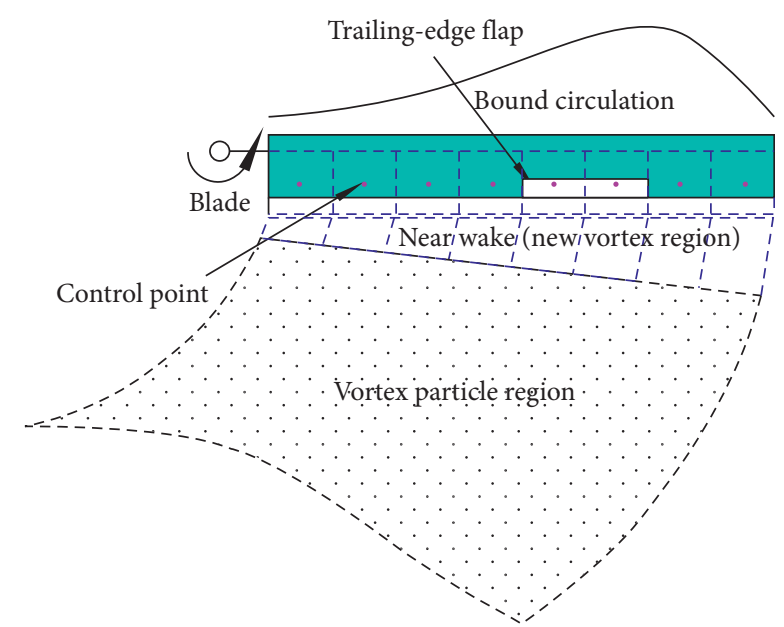

FIGURE 1: Solution diagram of the vortex lattice bound to the ECR blade surface and new vortex particle.

The unit normal vector of the blade surface at the control point is determined by the geometric incidence of the blade segment. Because the trailing edge flap is introduced, the effect of flap deflection and flap overhang on the unit normal vector of the surface can be represented by using the equivalent blade angle of incidence [15]:

$$
\alpha_{\mathrm{eff}}=\alpha+\frac{1}{\pi}\left(T_{10}-l T_{21}\right) \delta
$$

where $\alpha_{\text {eff }}$ is the equivalent incidence of the flap segment airfoil for the ECR blade, $\alpha$ is the geometric incidence of the flap segment basic airfoil, $\delta$ is the flap deflection angle and downward deflection is positive, $l$ is the nondimensional flap overhang corresponding to the semichord length of the airfoil, $T_{10}$ and $T_{21}$ are constants related to the flap chordwise location. For detailed expressions, please refer to reference [41].

After the blade-bound vortex distribution is obtained, the near-wake vortex lattice of each blade segment at the current azimuth is replaced by an equivalent vortex particle. The source term in formula (3) can be written as

$$
S=-\frac{\mathrm{d} \omega_{\mathrm{b}}}{\mathrm{d} t}+\mathbf{u}_{\mathrm{b}} \nabla \cdot \boldsymbol{\omega}_{\mathrm{b}}
$$

where $\boldsymbol{\omega}_{\mathrm{b}}$ is the bound vorticity of the blade segment and $\mathbf{u}_{\mathrm{b}}$ is the velocity of the blade segment with respect to the fluid.

Once the iterations of the rotor wake and bound circulation converge, the unsteady airloads of the airfoil and the incremental airloads caused by trailing-edge flap motion are obtained by using the Leishman-Beddoes model [39] and the Hariharan-Leishman model [40], respectively.

2.4. BVI Noise Prediction. The evaluation of ECR aerodynamic noise is based on the $\mathrm{FW}-\mathrm{H}$ equations derived from Lighthill's acoustic analogy approach, which contains monopole sources noise (thickness noise), dipole sources noise (loading noise), and quadrupole sources noise. Since the BVI event usually occurs in low-speed descent flight, the contribution of the quadrupole noise to the whole acoustic field is very small, so Farassat's formulation 1A obtained by ignoring the quadrupole noise term in the FW$\mathrm{H}$ equation is used in the noise prediction in this paper. The acoustic pressure $p^{\prime}(\mathbf{x}, t)$ at an observation point can be written as

$$
p^{\prime}(\mathbf{x}, t)=p_{\mathrm{t}}^{\prime}(\mathbf{x}, t)+p_{\mathrm{l}}^{\prime}(\mathbf{x}, t)
$$

where $p_{\mathrm{t}}^{\prime}(\mathbf{x}, t)$ and $p_{1}^{\prime}(\mathbf{x}, t)$ are the thickness noise and the loading noise, respectively. The thickness noise can be written as

$$
\begin{aligned}
4 \pi p_{\mathrm{t}}^{\prime}(\mathbf{x}, t)= & \int_{f=0}\left[\frac{\rho_{0} \dot{u}_{\mathrm{n}}}{r\left(1-M_{\mathrm{r}}\right)}\right]_{\mathrm{ret}} \mathrm{d} s \\
& +\int_{f=0}\left[\frac{\rho_{0} u_{\mathrm{n}}\left(r \dot{M}_{\mathrm{r}}+c_{0} M_{\mathrm{r}}-c_{0} M^{2}\right)}{r^{2}\left(1-M_{\mathrm{r}}\right)^{3}}\right]_{\mathrm{ret}} \mathrm{d} s,
\end{aligned}
$$

and the loading noise can be written as

$$
\begin{aligned}
4 \pi p_{1}^{\prime}(\mathbf{x}, t)= & \frac{1}{c_{0}} \int_{f=0}\left[\frac{i_{\mathrm{r}}}{r\left(1-M_{\mathrm{r}}\right)^{2}}\right]_{\mathrm{ret}} \mathrm{d} s+\int_{f=0}\left[\frac{l_{\mathrm{r}}-l_{\mathrm{M}}}{r^{2}\left(1-M_{\mathrm{r}}\right)^{2}}\right]_{\mathrm{ret}} \mathrm{d} s \\
& +\frac{1}{c_{0}} \int_{f=0}\left[\frac{l_{\mathrm{r}}\left(r \dot{M}_{\mathrm{r}}+c_{0} M_{\mathrm{r}}-c_{0} M^{2}\right)}{r^{2}\left(1-M_{\mathrm{r}}\right)^{3}}\right]_{\mathrm{ret}} \mathrm{d} s,
\end{aligned}
$$

where $l$ is the local force on the fluid per unit area, $c_{0}$ is the speed of sound in undisturbed flow, $r$ is the noise radiation distance, and $M$ is the surface Mach number. Besides, in these equations a dot over a variable and the subscript $n, r$, and $\mathrm{M}$ refer to the dot product with the unit normal vector, the unit radiation vector, and the surface velocity vector normalized by the speed of sound, respectively. The subscript ret indicates that the integrand should be evaluated at the retarded time on the blade surface $f=0$. Since the Weissinger- $\mathrm{L}$ model is used in this paper, the aerodynamic force contributed by each blade panel is equivalent to a compact acoustic source at the control point of each panel. It should be pointed out that the rotor BVI noise is essentially a loading noise. In the case of BVI occurrence, the thickness noise tends to be negligible and the acoustic pressure is dominated by the loading noise.

The flowchart of BVI-induced airloads and noise prediction of ECR is shown in Figure 2. Firstly, based on VVPM, the Weissinger-L model and the difference-based trim strategy [42], the blade pitch angle caused by flap deflection and the blade airloads are obtained. Then, according to the flight condition, the flap-deflection schedule, blade pitch angle and blade airloads, the time history of acoustic pressure at an observation point is obtained by using FW-H equations. Finally, the sound pressure level (SPL) of specific frequency noise is obtained by using the Fourier transform.

\section{Results and Analysis}

3.1. Validation of BVI Airloads and Noise Computational Model. Currently, there are few ECR-related tests, and 


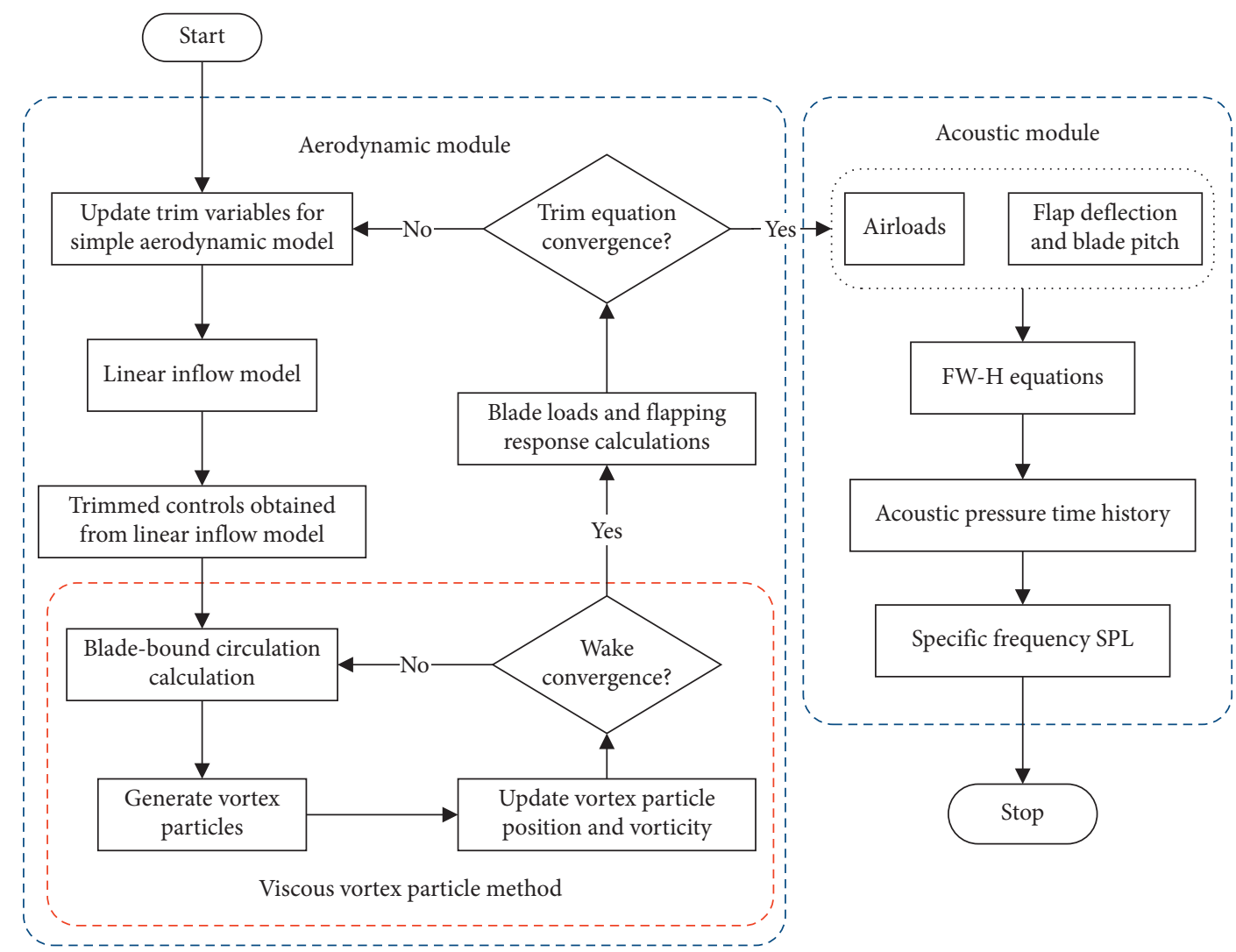

FIGURE 2: Schematic flowchart of BVI airloads and noise prediction of ECR.

there is no literature regarding BVI-induced airloads and noise of ECR. Therefore, in this section, the HART II test $\mathrm{BL}$ case is used as an example to validate the present BVIinduced airloads and noise-predicted model by comparing the blade airloads and the noise footprint on a horizontal plane below the rotor hub. The HART II test rotor has four hingeless blades with a rectangular tip, and the radius, chord, linear twist, and precone of the rotor are $2 \mathrm{~m}$, $0.121 \mathrm{~m},-8^{\circ}$, and $2.5^{\circ}$, respectively. The rotor was operated at an advance ratio of 0.151 , with a rotor shaft angle of attack of $5.3^{\circ}$. The wind tunnel interference angle is $-0.8^{\circ}$, which means that the effective angle of attack is reduced from $5.3^{\circ}$ to approximately $4.5^{\circ}$. During the test, the rotor had a thrust coefficient of 0.00457 . Other relevant parameters of the test are listed in reference [26]. The rotor was trimmed to the experimental thrust coefficient and to zero rolling and pitching moments about the rotor hub in the present simulation.

The comparison of the predicted and experimental section normal force $C_{n} M^{2}$ at the $87 \%$ span location is given in Figure 3. The predicted result in reference [30] is also shown in the figure, which is obtained by using the LBM. As shown, the present predicted airloads fluctuates dramatically around $50^{\circ}$ azimuth angle on the advancing side and around $300^{\circ}$ on the retreating side, which compares well with the experimental data. The accuracy of the present prediction on the advancing side is comparable to the LBM results, and the present results on the retreating side agree better with the experimental measurements than the LBM results, whose

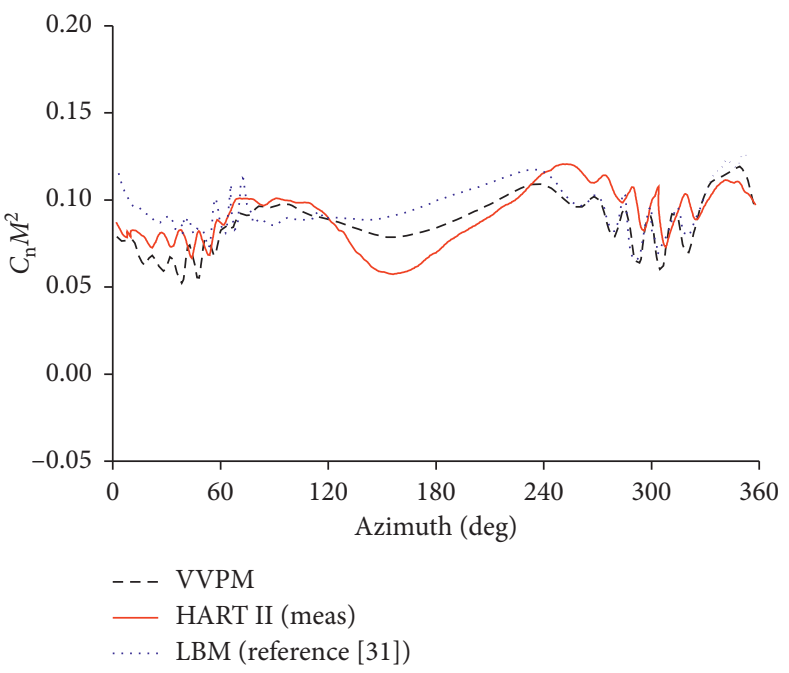

Figure 3: Normal force $\left(C_{\mathrm{n}} M^{2}\right)$ predictions at $87 \%$ radial station.

results have large spurious fluctuations between $60^{\circ}$ and $90^{\circ}$ in the azimuth. However, since the elastic deformation of the blade is not considered in the analytical model, there are some discrepancies between the predicted airloads and the experimental data. Especially around $180^{\circ}$ azimuth angle, the rigid blade model does not consider the reduction of the angle of attack caused by torsional deformation, the predicted airloads is obviously higher than the experimental data. 


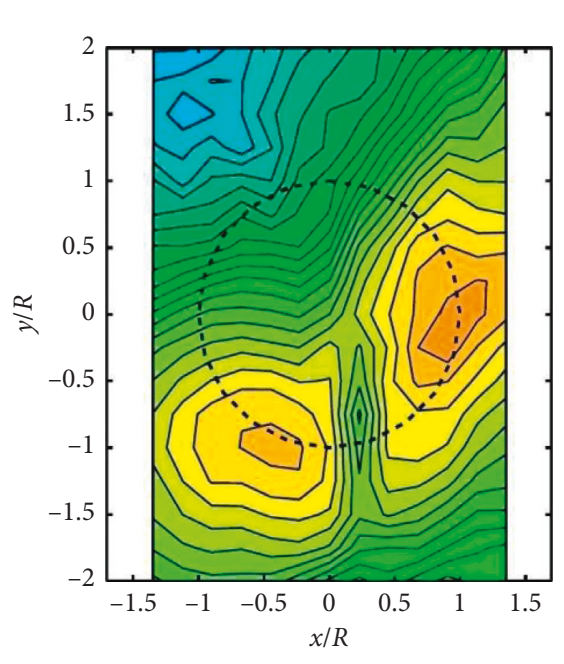

(a)

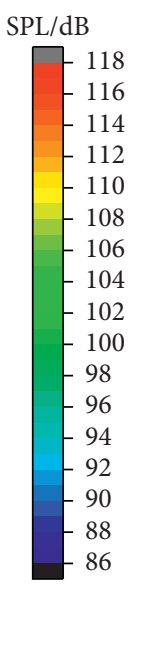

FIgUre 4: Predicted and measured SPL noise contours (6th-40th BPF). (a) Measured; (b) predicted.

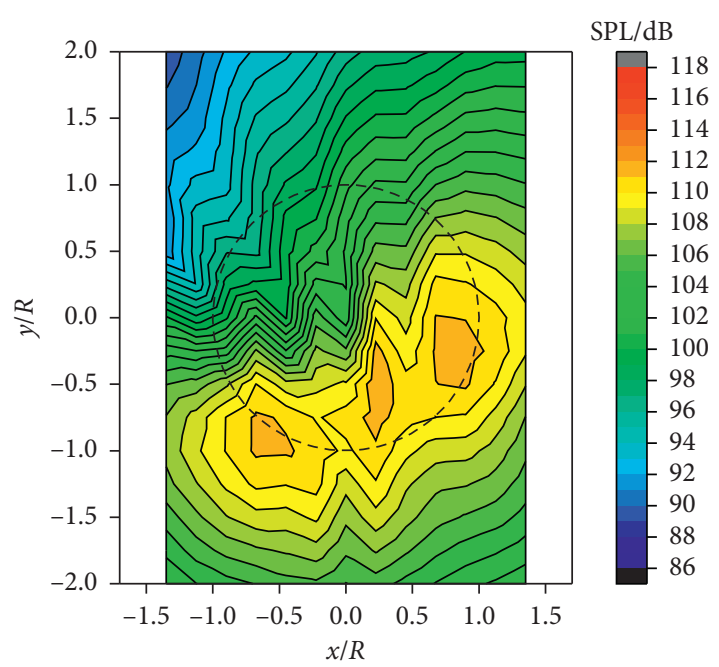

(b)
A comparison between the predicted and measured BVI noise footprint on a horizontal plane which placed $1.1075 R$ below the rotor hub is shown in Figure 4. The noise level is filtered to include the frequencies only from the 6th to the 40th blade passage frequency (BPF), which are mainly associated with the BVI event. The meaning of the circle drawn with dashed line is the projection of the rotor tip path. The simulation results show that two distinct radiation lobes appear in the advancing and retreating sides, which is the same with the experimental data obtained from the HART II test. The location and magnitude of the SPL maximum on the retreating side is an agreement with the measurements very well, while the magnitude of the SPL maximum on the advancing side is slightly underestimated by $2-3 \mathrm{~dB}$, and the location of the SPL maximum on the advancing side is further to the rear of the rotor. In addition, the predicted BVI noise in the center and to the rear of the rotor disc is larger than the measured data because the effect of the fuselage on the absorption and scattering of noise is not considered in the simulation.

In the preceding context, the prediction model of BVI airloads and noise has been validated with the HART II test database. The compared results show that the present model is capable of predicting the rotor BVI-induced airloads and noise.

3.2. Analysis of BVI Airloads and Noise Characteristics of ECR. In this section, the HART II test rotor is used as a reference to rebuilding an ECR, and the analysis of the BVI-induced airloads and noise of ECR is performed under the HART II test BL condition which has been described in Section 3.1. The main parameters of the sample ECR are listed in Table 1. Other parameters of the ECR are identical to those of the HART II test rotor.

As mentioned in the previous section, ECR primary control is implemented via flap motion. In this paper, the
TABLE 1: The main parameters of a sample ECR.

\begin{tabular}{lc}
\hline Pre-index angle & $6^{\circ}$ \\
Blade-root torsional stiffness & $95 \mathrm{Nm} / \mathrm{rad}$ \\
Flap chord & $0.03025 \mathrm{~m}$ \\
Flap spanwise length & $0.4 \mathrm{~m}$ \\
Flap midspan location & $1.4 \mathrm{~m}$ \\
Nondimensional flap overhang & 0.125 \\
\hline
\end{tabular}

TABLE 2: The trimming results of the flap deflection.

\begin{tabular}{lccc}
\hline Flap deflection & $\delta_{0}$ & $\delta_{c}$ & $\delta_{s}$ \\
\hline Value & $3.27^{\circ}$ & $-4.65^{\circ}$ & $0.96^{\circ}$ \\
\hline
\end{tabular}

ECR control variables include the flap collective deflection $\delta_{0}$, flap lateral cyclic deflection $\delta_{c}$, and flap longitudinal cyclic deflection $\delta_{s}$. The trimming results of the flap deflections of the ECR are listed in Table 2. It is emphasized again that the flap downward deflection is positive. Because of the large pre-index angle, the flap collective deflection $\delta_{0}$ is positive to achieve the target thrust coefficient by reducing the blade collective pitch. The flap lateral cyclic deflection $\delta_{c}$ is positive, because of the upwash and downwash on the front and rear parts, respectively, of the rotor disk. In addition, the longitudinal cyclic deflection $\delta_{s}$ is pretty small, because the velocities of the blade at the advancing side and retreating side versus air are different, the flap collective deflection generates cyclic pitch moment on the blade, namely the ECR "collective pitch-cyclic pitch effect."

Figure 5 compares the blade-pitch angle variation with azimuth between the ECR and the conventional rotor (HART II test rotor). It should be noted that instead of the measured data, the predicted results of HART II test rotor are adopted to compare with the predicted ECR results in this paper. The comparison results show that the minimum blade pitch angle of the ECR appears around $120^{\circ}$ 


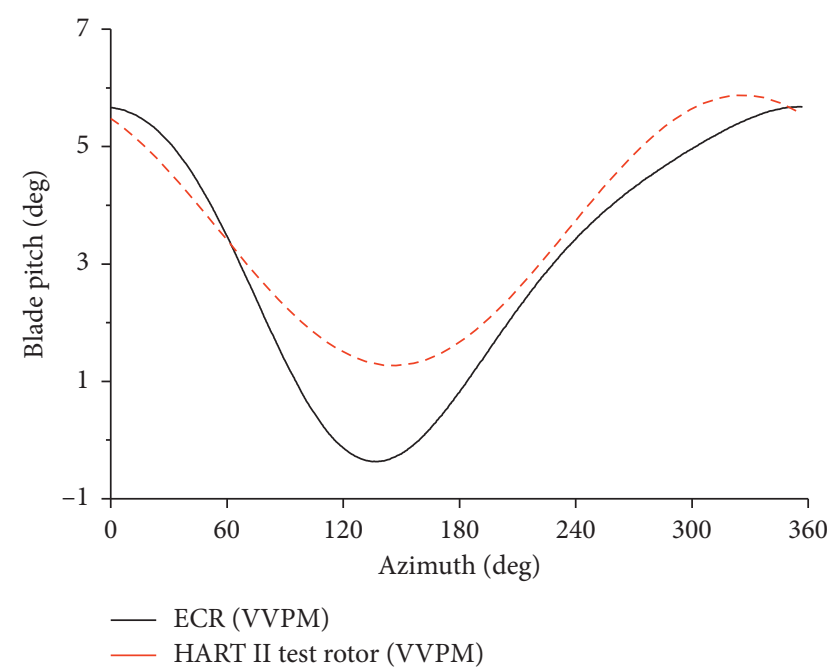

FIGURE 5: Variation of blade pitch of the ECR and the conventional rotor versus azimuth angle.

azimuth angle, which is almost the same as that of the conventional rotor. However, because of the additional lift generated by the flap deflection, there are some differences between the ECR and conventional rotors blade pitch angle magnitude. Especially around the $120^{\circ}$ azimuth angle, the pitch angle of ECR is significantly smaller than that of the conventional rotor, which is caused by the large downward deflection of the flap around the azimuth angle.

The deflection of the flaps changes the load distribution along the blade span, which leads to the difference between the wake vorticity distribution of the ECR and that of the conventional rotor. Figure 6 shows the vorticity isosurfaces diagrams of the ECR wake and the conventional rotor wake under the HART II test BL condition. To further illustrate the direction of the vorticity, the vorticity isosurfaces diagram is colored by the vorticity value in the $x$-direction. Because of the small blade pitch angle, there are no strong tip vortices between the $90^{\circ}$ to the $180^{\circ}$ azimuth angle on the ECR advancing side. In fact, the predicted result illustrates the strong vortex is shed from the tip of the flap in the second quadrant because of the large flap deflection. Meanwhile, since the difference in the magnitude of the blade pitch angle on the retreating side between the ECR and the conventional rotor is very small, the wake vorticity structure on the retreating side is almost the same as that of the conventional rotor. In addition, both the ECR wake vortices and the conventional rotor wake vortices will rapidly roll up along the rotor-advancing and -retreating sides.

Because of the difference between the ECR and conventional rotor-wake structure on the advancing side, when the BVI occurs, the amplitude and location of the impulsive airloads of the ECR on the advancing side will be quite different from those of the conventional rotor. Figures 7 (a) and 7 (b) show the section normal force distribution of the ECR and conventional rotor, respectively. According to the reference [27], the highfrequency component (higher than 10/rev) of the section



(a)



(b)

Figure 6: Vorticity isosurfaces diagram of the wake flow field for the ECR and conventional rotor. (a) ECR with $6^{\circ}$ pre-index angle; (b) conventional rotor.

normal force is almost exclusively due to the BVI. To further illustrate the difference between the BVI-induced airloads of the ECR and the conventional rotor, the high harmonic section normal force $(>10 / \mathrm{rev})$ at the $70 \%$ (blade segment with flap) and $87 \%$ (blade segment without flap) span locations are shown in the Figures 7 (c) and 7(d), respectively. Owing to the flap motion, the section normal force distribution of the ECR is quite different from that of the conventional rotor. But the BVI-induced impulsive airloads can be also observed on the ECRadvancing side and retreating side. Furthermore, the amplitude of the ECR impulsive airloads on the advancing side is greater than that of the conventional rotor at both $70 \%$ and $87 \%$ span locations, and the blade spanwise range of the ECR BVI occurrence on the advancing side is smaller than that of the conventional rotor, especially around the $60^{\circ}$ azimuth angle. At the $70 \%$ span location, the amplitude of ECR impulsive airloads around the $60^{\circ}$ azimuth angle is still greater than that of the conventional rotor, but at $87 \%$ span location, the amplitude of the ECR impulsive airloads around the $60^{\circ}$ azimuth angle is significantly less than that of the conventional rotor. As mentioned above, the BVI event of the ECR on the advancing side is mainly caused by the interaction between the flap tip vortices and the blades. The theoretical interaction region of ECR and 


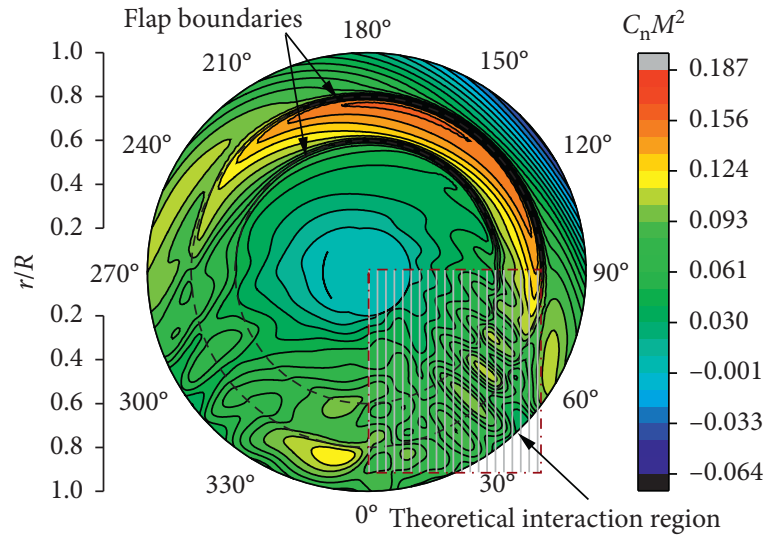

(a)

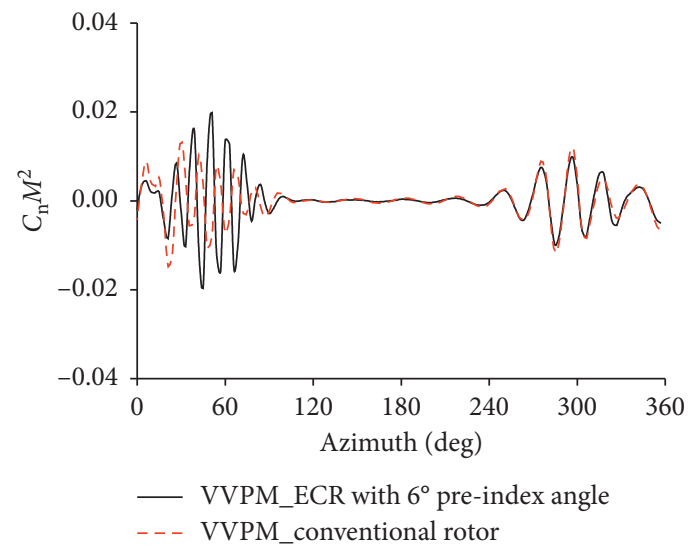

(c)

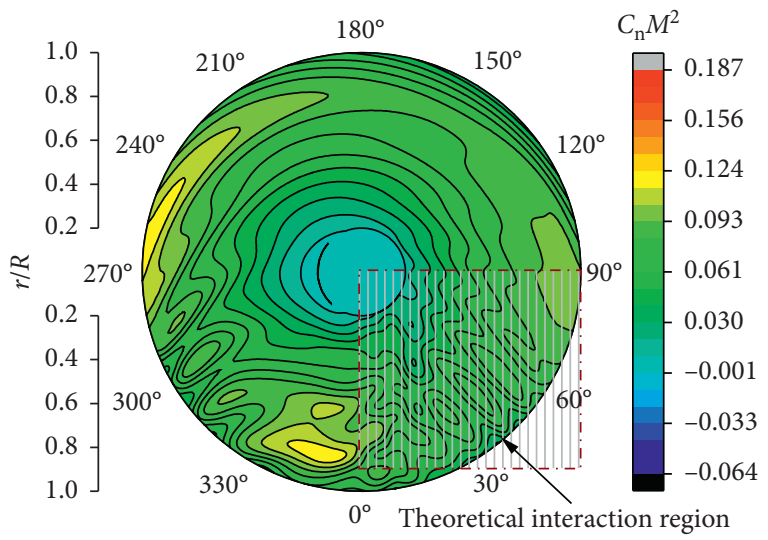

(b)

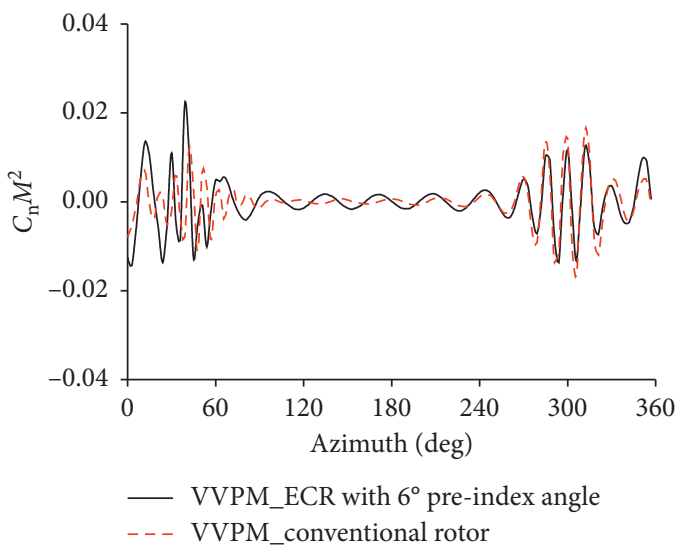

(d)

FIGURE 7: BVI-induced airloads of the ECR and conventional rotor. (a) Disc airloads distribution of the ECR with $6^{\circ}$ pre-index angle; (b) disc airloads distribution of the conventional rotor; (c) high-frequency (>10/rev) section normal force $C_{\mathrm{n}} M^{2}$ at $70 \%$ span location; (d) highfrequency $(>10 / \mathrm{rev})$ section normal force $C_{\mathrm{n}} M^{2}$ at $87 \%$ span location.

conventional rotor on the advancing side are also shown in Figures 7(a) and 7(b), respectively. The difference in the theoretical interaction region between the ECR and the conventional rotor is the main reason for the different width of the BVI occurrence. On the retreating side, the amplitude of the ECR impulsive airloads at both $70 \%$ and $87 \%$ radial station are slightly less than those of the conventional rotor. On the other hand, there are slight discrepancies of the phase of the impulsive airloads on the advancing side between the ECR and the conventional rotor, while the phase of the impulsive airloads on the retreating side is almost the same.

Based on the predicted BVI-induced airloads of the ECR, the BVI noise footprints of the ECR and the conventional rotor on a horizontal plane which placed $1.1075 R$ below the rotor hub are given in Figure 8. The noise levels are still filtered to include only the frequencies between 6-40 times BPF. The magnitude of the SPL maximum on the advancing side of the ECR is $4 \mathrm{~dB}$ higher than that of the conventional rotor, which is caused by the larger ECR airloads on the advancing side. The magnitude of the SPL maximum on the retreating side and the center of the ECR are $3 \mathrm{~dB}$ less than that of the conventional rotor, although the amplitude of the
BVI-induced airloads on the retreating side of the ECR is just slightly less than that of the conventional rotor.

\subsection{Effect of ECR Pre-Index Angle on BVI-Induced Airloads} and Noise. Trimming result of ECR flap deflection varies greatly with different blade pre-index angles. Owing to the flap motion, the ECR wake vorticity field is more complex than that of the conventional rotor. The BVI-induced airloads and noise are closely related to the wake structure of the rotor. Therefore, the influence of pre-index angle on the BVI-induced airloads and noise of the ECR is explored in this section.

Table 3 shows the trimmed flap deflection of the ECR with different pre-index angles under the HART II test BL condition. With the increase in pre-index angle, the ECR flap collective deflection $\delta_{0}$ gradually deflects from upward to downward, while the flap lateral cyclic deflection $\delta_{c}$ does not significantly change. The flap longitudinal cyclic deflection $\delta_{s}$ varies significantly with increasing pre-index angle, which is the result from the collective pitch-cyclic pitch effect of the ECR.

The predicted airloads of the ECR with $4^{\circ}$ pre-index angle and $8^{\circ}$ pre-index angle are shown in Figures 9 and 10, 




(a)

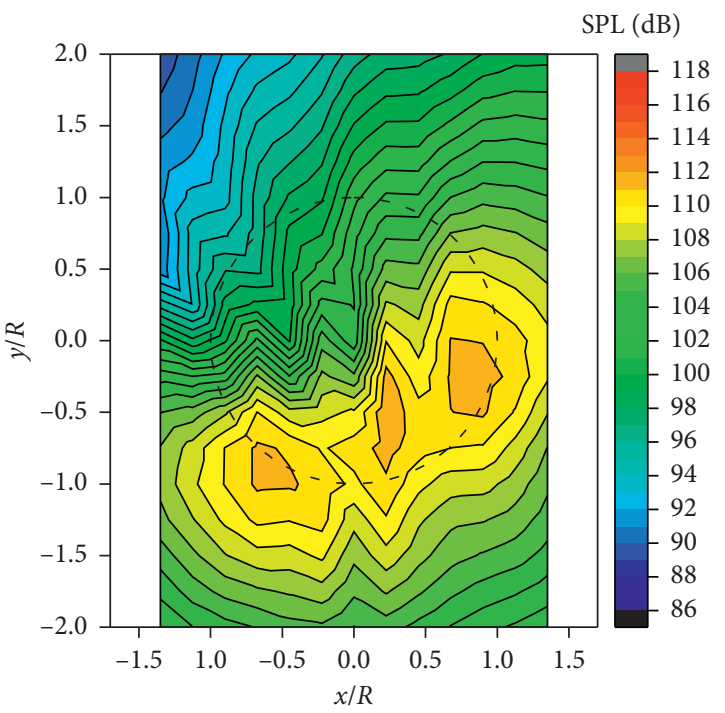

(b)

Figure 8: Predicted SPL noise (6th-40th BPF) contours. (a) ECR with $6^{\circ}$ pre-index angle; (b) conventional rotor.

TABLE 3: The trimming results of the flap deflection of the ECR with different pre-index angles.

\begin{tabular}{lccr}
\hline Pre-index angle & $\delta_{0}$ & $\delta_{c}$ & $\delta_{s}$ \\
\hline $4^{\circ}$ & $-1.97^{\circ}$ & $-4.77^{\circ}$ & $4.12^{\circ}$ \\
$6^{\circ}$ & $3.27^{\circ}$ & $-4.65^{\circ}$ & $0.96^{\circ}$ \\
$8^{\circ}$ & $8.43^{\circ}$ & $-4.54^{\circ}$ & $-2.1^{\circ}$ \\
\hline
\end{tabular}



(a)

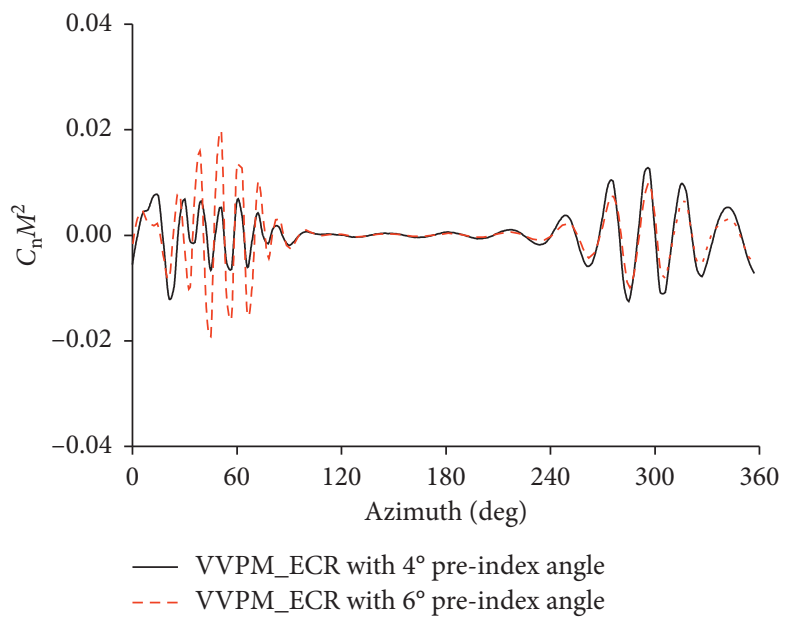

(b)

Figure 9: Continued. 


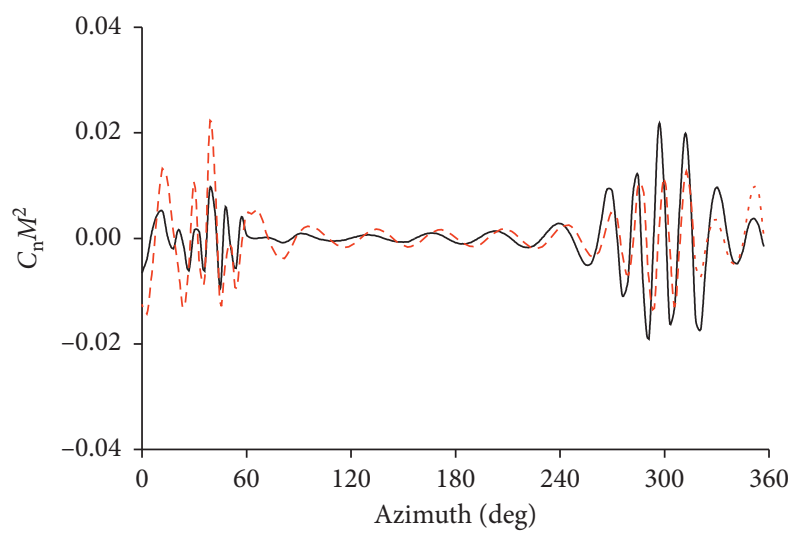

_ VVPM_ECR with $4^{\circ}$ pre-index angle

—VVPM_ECR with $6^{\circ}$ pre-index angle

(c)

Figure 9: BVI airloads of ECR with $4^{\circ}$ pre-index angle. (a) Disc airloads distribution; (b) high-frequency ( $>10 /$ rev) section normal force $C_{\mathrm{n}} M^{2}$ at $70 \%$ span location; (c) high-frequency (>10/rev) section normal force $C_{\mathrm{n}} M^{2}$ at $87 \%$ span location.

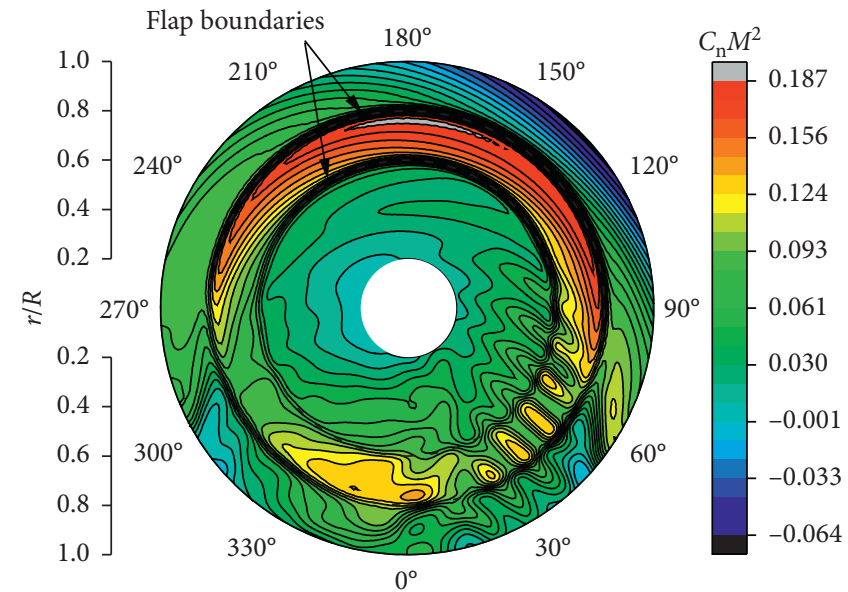

(a)



- VVPM_ECR with $8^{\circ}$ pre-index angle - - - VVPM_ECR with $6^{\circ}$ pre-index angle

(b)

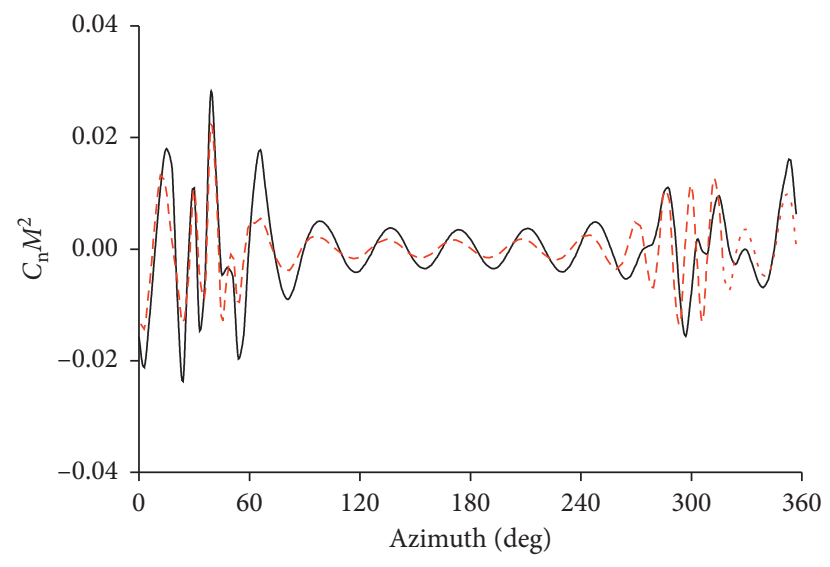

- VVPM_ECR with $8^{\circ}$ pre-index angle VVPM_ECR with $6^{\circ}$ pre-index angle

(c)

FIGURE 10: BVI airloads of ECR with $8^{\circ}$ pre-index angle. (a) Disc airloads distribution; (b) high-frequency ( $>10 /$ rev) section normal force $C_{\mathrm{n}} M^{2}$ at $70 \%$ span location; (c) high-frequency (>10/rev) section normal force $C_{\mathrm{n}} M^{2}$ at $87 \%$ span location. 
respectively. The predicted section normal force of the ECR with $6^{\circ}$ pre-index angle is also shown in the figures for comparison. It can be seen that the airloads distribution of the ECR with different pre-index angles presents a significant difference. Due to the large downward flap deflection, the lift maximum of the ECR with $8^{\circ}$ pre-index angle is located on the blade section with the flap. When the ECR pre-index angle is $4^{\circ}$, the flap upward deflection on the retreating side reduces the local airloads on the blade section with the flap. However, whether the pre-index angle is $4^{\circ}$ or $8^{\circ}$, the blade spanwise range of the BVI occurrence of the ECR on the advancing side is almost the same as that of the ECR with $6^{\circ}$ pre-index angle, which demonstrates the BVI events of ECR with $4^{\circ}$ and $8^{\circ}$ on the advancing side are still caused by the interaction between the flap tip vortices and the blades. The amplitude of the impulsive airloads of the ECR on the advancing side is increased with the increase in pre-index angle, while the amplitude of the impulsive airloads of the ECR on the retreating side is decreased significantly. Besides, the phases of the BVIinduced airloads of the ECR with different pre-index angles are almost identical.

The predicted BVI noise footprint of ECR with $4^{\circ}$ preindex angle on the carpet of microphones are given in Figures 11(a) and 11(b) depicts the contour plot of the differences of the noise predictions obtained by considering the ECR with $4^{\circ}$ pre-index angle and $6^{\circ}$ pre-index angle. The numerical results for the ECR with $8^{\circ}$ pre-index angle are shown in Figure 12. The figures illustrate that with the increasing of pre-index angle, the magnitude of the SPL maximum on the advancing side of the ECR increases significantly. The magnitude of the advancing side SPL maximum is increased about $6 \mathrm{~dB}$ while the pre-index angle increases from $4^{\circ}$ to $8^{\circ}$. However, with the increasing of preindex angle, the magnitude of the SPL maximum on the retreating side of the ECR decreases. Especially when the pre-index angle is $8^{\circ}$, the radiation lobe in the retreating side is almost disappeared.

Finally, in order to explore the reason for the difference in BVI-induced airloads and noise among the ECR with different pre-index angles, the ECR wake contours on two lateral planes. The one at $y=0.6 R$ on the advancing side and the other at $y=-0.7 R$ on the retreating side are investigated (see Figure 13).

Figures 14-16 show the ECR wake vorticity contour over the advancing-side plane and retreating-side plane. The contours show that with the increasing of pre-index angle, the strength of wake vorticity on the advancing side of the ECR increases, while the strength of the wake vorticity on the retreating side decreases. In addition, among the ECR with three different pre-index angles, the difference in the position of the vortex core is very small. The investigation results demonstrate that the strength of wake vorticity is the main reason for the difference in the BVI-induced airloads and noise among the ECR with

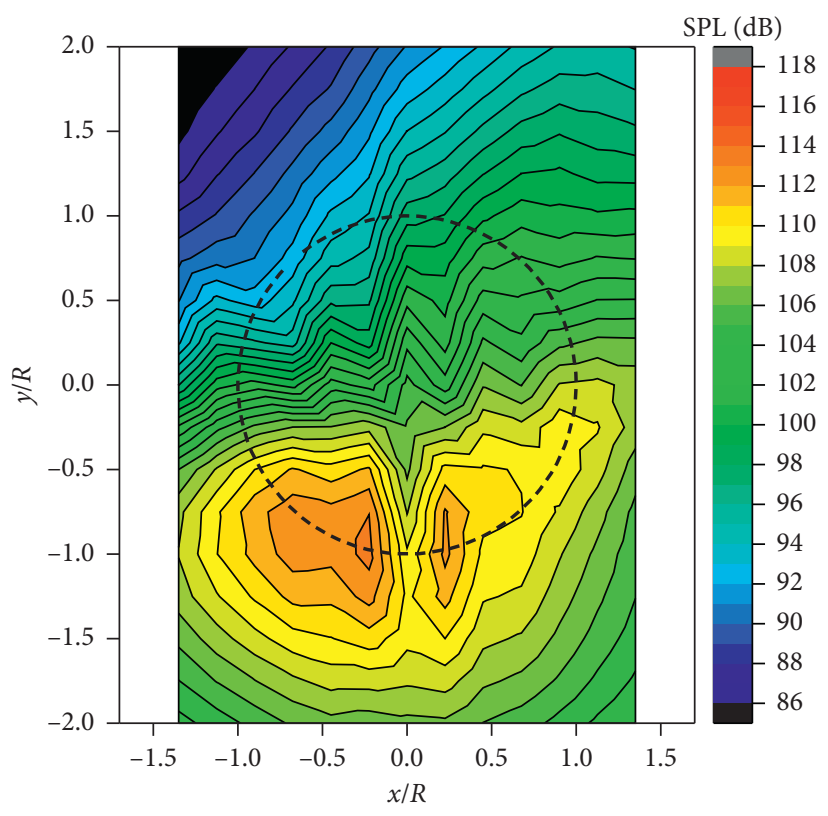

(a)

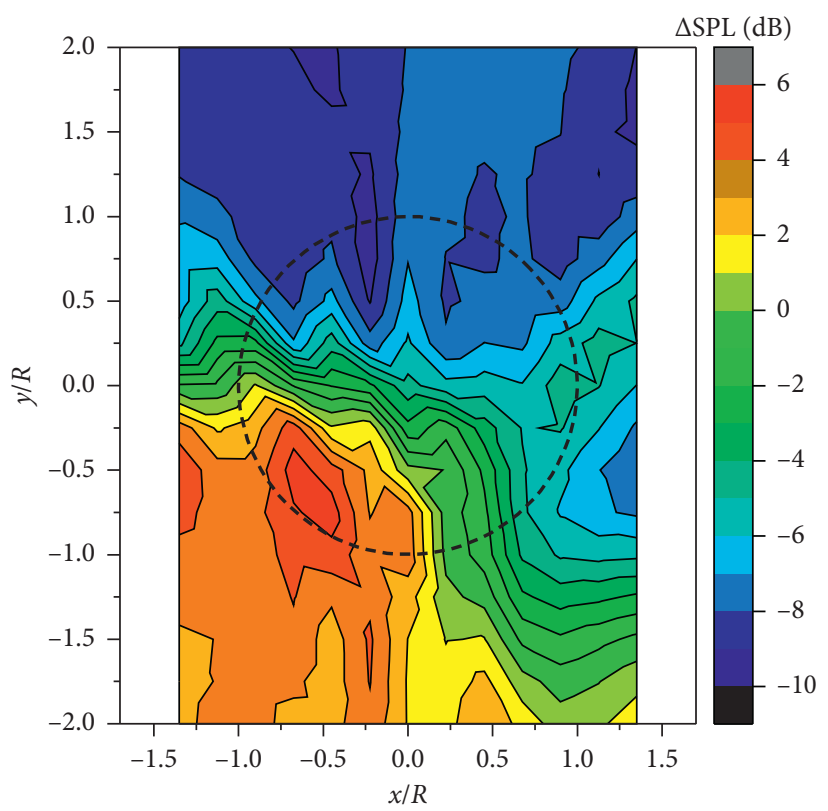

(b)

Figure 11: Midfrequency (6th-40th) BVI noise directivity pattern of the ECR with $4^{\circ}$ pre-index angle. (a) Predicted SPL noise contours; (b) difference of SPL between ECR with $4^{\circ}$ and $6^{\circ}$ preindex angle.

different pre-index angles. Incidentally, because of the BVI-induced airloads and noise varying with the preindex angle, the nonharmonic active control method can be used to reduce the strong BVI event on the advancing side or retreating side of the ECR with a specific pre-index angle. 


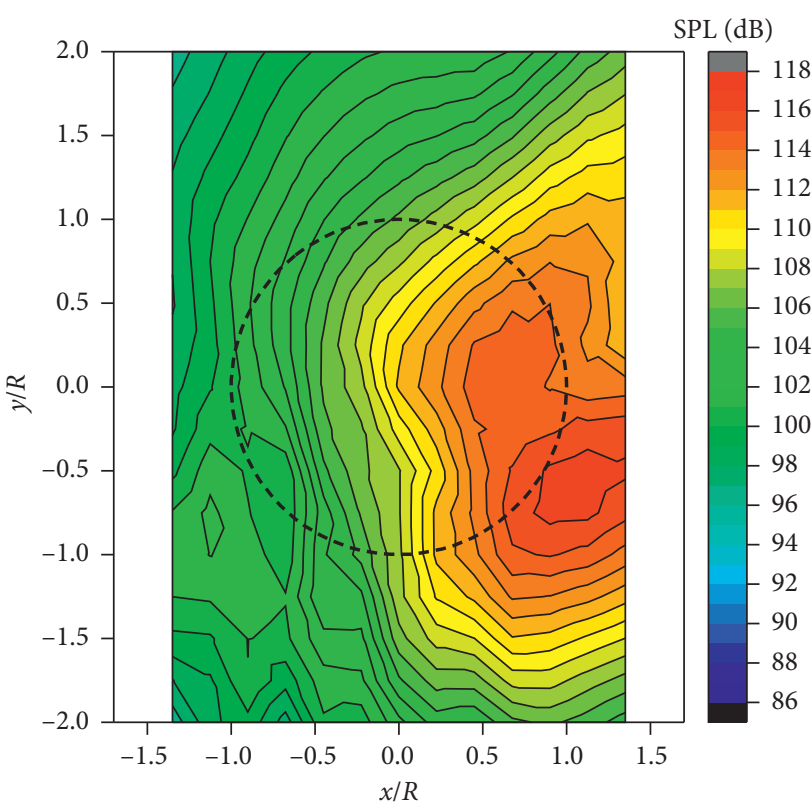

(a)

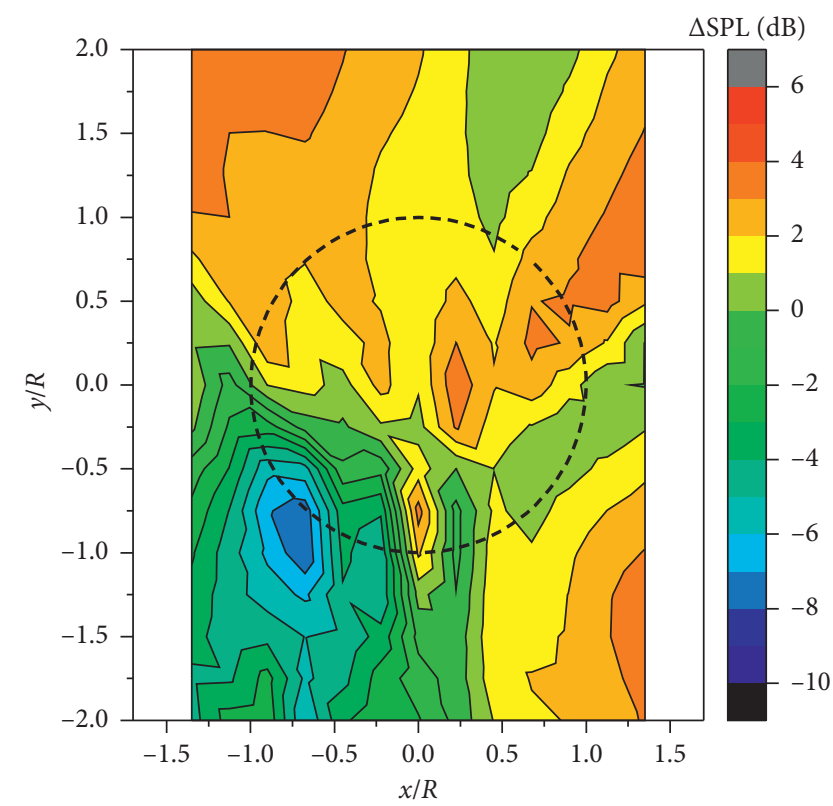

(b)

FIgURE 12: Midfrequency (6th-40th) BVI noise directivity pattern of the ECR with $8^{\circ}$ pre-index angle. (a) Predicted SPL noise contours; (b) difference of SPL between ECR with $8^{\circ}$ and $6^{\circ}$ pre-index angle.

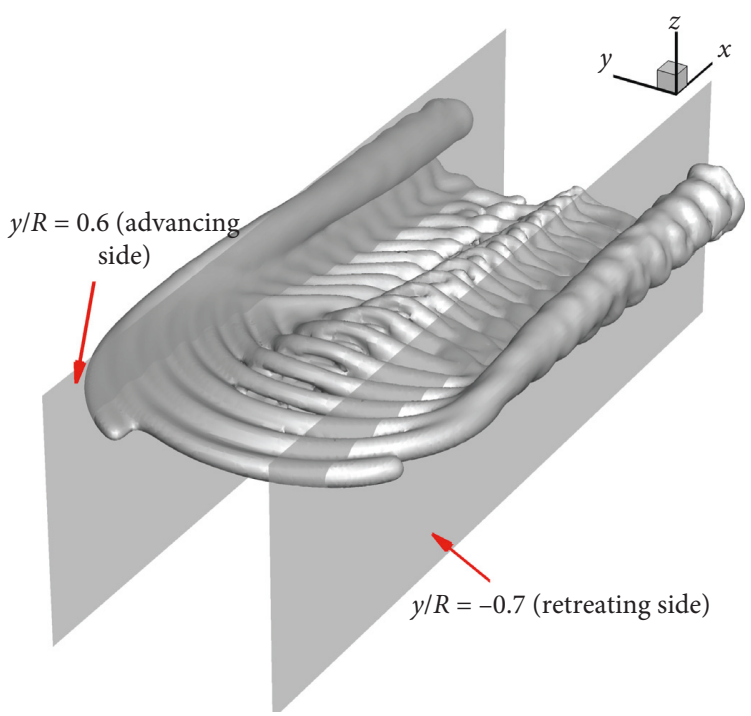

FIGURE 13: Longitudinal planes for investigation of wake vorticity of ECR.



(a)



(b)

FIgURE 14: Predicted wake vorticity contour of ECR with $4^{\circ}$ pre-index angle over the longitudinal planes. (a) Plane at $y=0.6 R$ on the advancing side; (b) Plane at $y=-0.7 R$ on the retreating side. 


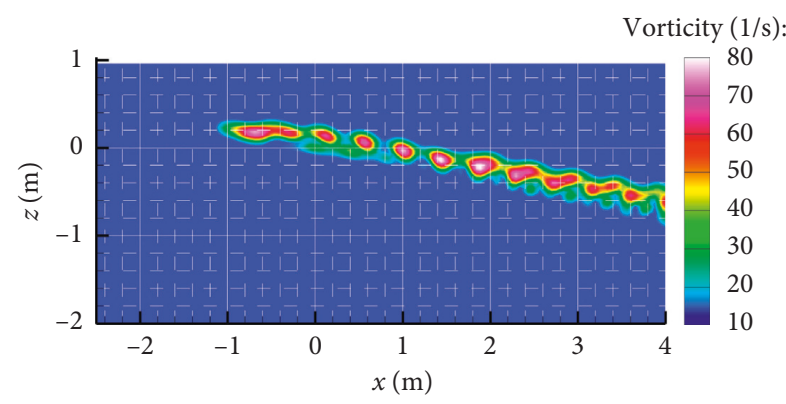

(a)

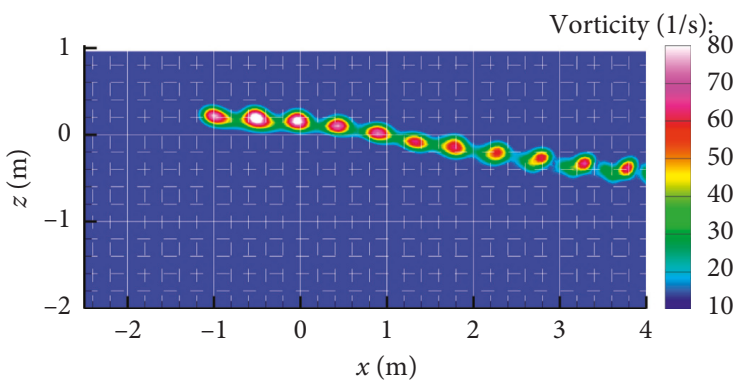

(b)

FIgURe 15: Predicted wake vorticity contour of ECR with $6^{\circ}$ pre-index angle over the longitudinal planes. (a) Plane at $y=0.6 R$ on the advancing side; (b) plane at $y=-0.7 R$ on the retreating side.



(a)

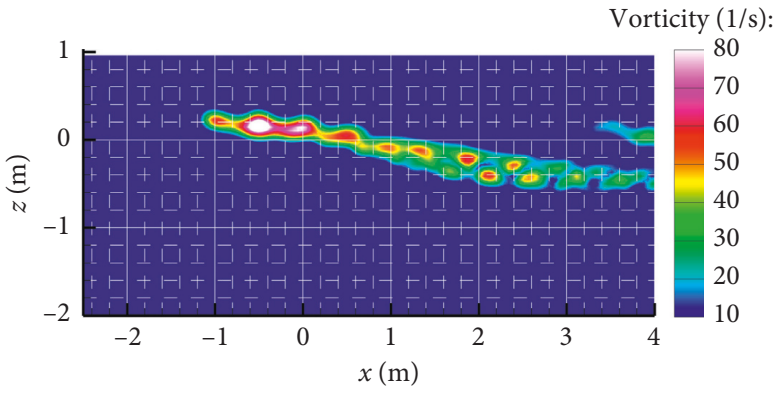

(b)

FIGURE 16: Predicted wake vorticity contour of ECR with $8^{\circ}$ pre-index angle over the longitudinal planes. (a) Plane at $y=0.6 R$ on the advancing side; (b) plane at $y=-0.7 R$ on the retreating side.

\section{Conclusion}

In this paper, based on the viscous vortex particle method, ECR blade pitch-movement equation, and Weissinger-L lifting surface model, a predicted model for ECR BVI-induced airloads is established. In the model, the effects of flap deflection on the blade bound vortex circulation distribution are considered by using the equivalent angle of incidence. The flap deflection induced blade pitch movement is obtained by solving the ECR blade pitch movement equation via the Runge-Kutta fourth-order method. On this basis, BVI noise radiation of an ECR is evaluated using a postprocessor for the blade airloads that implements Farassat's formulation $1 \mathrm{~A}$ of the FW-H equation. The predicted model was validated against the well known HART II test database. Furthermore, the aerodynamic and acoustic characteristics of a sample ECR in BVI conditions are analyzed, and a study was performed to examine the effect of the pre-index angle on the BVI-induced airloads and noise. The following conclusions have been drawn from the present study:

(1) The BVI-induced airloads predicted model established in this paper is capable of accurately predicting the impulsive airloads which is induced by the interactions between the blades and the vortices in the wake. In addition, the magnitude of the predicted SPL maximum on the retreating side is an agreement with the measurements very well, while the magnitude of the SPL maximum on the advancing side is slightly underestimated.

(2) Since the BVI event of the ECR on the advancing side is mainly caused by the interaction between the flap tip vortices and the blades, the blade spanwise range of the ECR BVI occurrence on the advancing side is smaller than that of the conventional rotor. Moreover, there are slight discrepancies in the phase of the advancing side impulsive airloads between the ECR and the conventional rotor. The magnitude of the SPL maximum on the advancing side of the ECR with $6^{\circ}$ pre-index angle is higher than that of the conventional rotor, which is consistent with the predicted airloads on the advancing side. The magnitude of the SPL maximum on the retreating side and the center of the ECR are less than that of the conventional rotor.

(3) The amplitude of the impulsive airloads of the ECR on the advancing side is increased with the increasing of the pre-index angle, while the amplitude of the impulsive airloads of the ECR on the retreating side is decreased significantly. The magnitude of the advancing side SPL maximum is increased about $6 \mathrm{~dB}$ while the pre-index angle increases from $4^{\circ}$ to $8^{\circ}$. Significantly, when the pre-index angle is $8^{\circ}$, the radiation lobe in the retreating side is almost disappeared. 
(4) The different strength of wake vorticity is the main reason for the difference in the BVI-induced airloads and noise among the ECR with different pre-index angles.

\section{Data Availability}

The data used to support the findings of this study are available from the corresponding author upon request.

\section{Conflicts of Interest}

The authors declare that they have no conflicts of interest.

\section{Acknowledgments}

The authors would like to thank the National Key Laboratory Foundation of China (No. 51375229) and National Key Laboratory of Rotorcraft Aeromechanics.

\section{References}

[1] T. Y. Su and Y. Lu, "Application of viscous vortex particle method to electrically controlled rotor wake and aerodynamic characteristics analysis," in Proceeding of the 7th Asian/ Australian Rotorcraft Forum, Jeju Island, Korea, October 2018.

[2] J. Shen and I. Chopra, "Swashplateless helicopter rotor with trailing-edge flaps," Journal of Aircraft, vol. 41, no. 2, pp. 208-214, 2004.

[3] S. R. Viswamurthy and R. Ganguli, "An optimization approach to vibration reduction in helicopter rotors with multiple active trailing edge flaps," Aerospace Science and Technology, vol. 8, no. 3, pp. 185-194, 2004.

[4] B. Roget and I. Chopra, "Wind-tunnel testing of rotor with individually controlled trailing-edge flaps for vibration reduction," Journal of Aircraft, vol. 45, no. 3, pp. 868-879, 2008.

[5] D. Patt, L. Liu, and P. P. Friedmann, "Simultaneous vibration and noise reduction in rotorcraft using aeroelastic simulation," Journal of the American Helicopter Society, vol. 51, no. 2, pp. 127-140, 2006.

[6] B. W. Sim, R. D. Janakiram, N. L. Barbely, and E. Solis, "Reduced in-plane, low frequency noise of an active flap rotor," in Proceedings of the 65th American Helicopter Society Annual Forum, Grapevine, TX, USA, May 2009.

[7] B. W. Sim, M. Potsdam, C. Kitaplioglu, P. LeMasurie, P. Lorber, and J. Andrews, "Localized, non-harmonic active flap motions for low frequency in-plane rotor noise reduction," in Proceedings of the 68th American Helicopter Society Annual Forum, Fort Worth, TX, USA, May 2012.

[8] L. Liu, P. P. Friedmann, I. Kim, and D. S. Bernstein, "Rotor performance enhancement and vibration reduction in presence of dynamic stall using actively controlled flaps," Journal of the American Helicopter Society, vol. 53, no. 4, pp. 338-350, 2008.

[9] K. Ravichandran, I. Chopra, B. E. Wake, and B. Hein, "Trailing edge flaps for rotor performance enhancement and vibration reduction," Journal of the American Helicopter Society, vol. 58, no. 2, pp. 1-13, 2013.

[10] W. Johnson, Rotorcraft Aeromechanics, Cambridge University Press, New York, NY, USA, 2013.

[11] R. A. Ormiston, "Aeroelastic considerations for rotorcraft primary control with on-blade elevons," in Proceedings of the 57th American Helicopter Society Annual Forum, Washington DC, USA, May 2001.

[12] J. Shen and I. Chopra, "Aeroelastic modeling of trailing-edgeflap helicopter rotors including actuator dynamics," Journal of Aircraft, vol. 41, no. 6, pp. 1465-1472, 2004.

[13] J. Falls, "Design and performance prediction of swashplateless helicopter rotor with trialing edge flaps and tabs," University of Maryland, College Park, MD, USA, Doctoral dissertation, 2010.

[14] J. Shen and I. Chopra, "A parametric design study for a swashplateless helicopter rotor with trailing-edge flaps," Journal of the American Helicopter Society, vol. 49, no. 1, pp. 43-53, 2004.

[15] Y. Lu and C. Wang, "Active control for performance enhancement of electrically controlled rotor," Chinese Journal of Aeronautics, vol. 28, no. 5, pp. 1494-1502, 2015.

[16] F. H. Schmitz and Y. H. Yu, "Helicopter impulsive noise: theoretical and experimental status," Journal of Sound and Vibration, vol. 109, no. 3, pp. 361-422, 1986.

[17] Y. H. Yu, C. Tung, J. Gallman et al., "Aerodynamics and acoustics of rotor blade-vortex interactions," Journal of Aircraft, vol. 32, no. 5, pp. 970-977, 1995.

[18] R. C. Strawn, E. P. N. Duque, and J. Ahmad, "Rotorcraft aeroacoustics computations with overset-grid CFD methods," Journal of the American Helicopter Society, vol. 44, no. 2, pp. 132-140, 1999.

[19] B. G. Wall, C. L. Burley, Y. Yu, H. Richard, K. Pengel, and P. Beaumier, "The HART II test-measurement of helicopter rotor wakes," Aerospace Science and Technology, vol. 8, no. 4, pp. 273-284, 2004.

[20] B. G. Wall, "2nd HHC aeroacoustic rotor test (HART II)-part I: test documentation," Institute Report IB 111-2003/31, DLR, Cologne, Germany, 2003.

[21] B. G. Wall and C. L. Burley, "2nd HHC aeroacoustic rotor test (HART II) - Part II: representative results,” Institute Report IB 111-2005, DLR, Cologne, Germany, 2005.

[22] J.-S. Park, S. N. Jung, S. H. Park, and Y. H. Yu, "Correlation study of a rotor in descending flight using DYMORE with a freewake model," Journal of Mechanical Science and Technology, vol. 24, no. 8, pp. 1583-1594, 2010.

[23] M. Gennaretti, G. Bernardini, J. Serafini, and G. Romani, "Rotorcraft comprehensive code assessment for blade-vortex interaction conditions," Aerospace Science and Technology, vol. 80, pp. 232-246, 2018.

[24] D. D. Broy, "HART II acoustic predictions using a coupled CFD/CSD method," in Proceedings of the 65th American Helicopter Society Annual Forum, Grapevine, TX, USA, May 2009.

[25] J. W. Lim and R. C. Strawn, "Prediction of HART II rotor BVI loading and wake system using CFD/CSD loose coupling," in Proceedings of the 45th AIAA Aerospace Sciences Meeting and Exhibit, Reno, NV, USA, January 2007.

[26] M. J. Smith, J. W. Lim, B. G. Wall et al., "An assessment of CFD/CSD prediction state of the art using the HART II international workshop data," in Proceedings of the 68th American Helicopter Society Annual Forum, Fort Worth, TX, USA, May 2012.

[27] M. E. Kelly and R. E. Brown, "Influence of blade aerodynamic model on the prediction of helicopter high-frequency airloads," Journal of Aircraft, vol. 48, no. 2, pp. 476-494, 2011.

[28] M. E. Kelly and R. E. Brown, "Influence of blade aerodynamic model on prediction of helicopter rotor aeroacoustic signatures," Journal of Aircraft, vol. 48, no. 3, pp. 1058-1083, 2011.

[29] Y. Y. Zhao, Y. J. Shi, and G. H. Xu, "Helicopter blade vortex interaction airload and noise prediction using coupling CFD/ VVPM method," Applied Sciences, vol. 7, no. 4, p. 381, 2017. 
[30] G. Romani and D. Casalino, "Rotorcraft blade-vortex interaction noise prediction using the Lattice-Boltzmann method," Aerospace Science and Technology, vol. 88, pp. 147-157, 2019.

[31] C. He and J. Zhao, "Modeling rotor wake dynamics with viscous vortex particle method," AIAA Journal, vol. 47, no. 4, pp. 902-915, 2009.

[32] J. F. Tan and H. W. Wang, "Simulating unsteady aerodynamics of helicopter rotor with panel/viscous vortex particle method," Aerospace Science and Technology, vol. 30, no. 1, pp. 255-268, 2013.

[33] Y. J. Shi, G. H. Xu, and P. Wei, "Rotor wake and flow analysis using a coupled Eulerian-Lagrangian method," Engineering Applications of Computational Fluid Mechanics, vol. 10, no. 1, pp. 386-404, 2016.

[34] P. Singh and P. P. Friedmann, "Application of vortex methods to coaxial rotor wake and load calculations in hover," Journal of Aircraft, vol. 55, no. 1, pp. 373-381, 2018.

[35] J. F. Tan, T. Y. Zhou, Y. M. Sun, and G. N. Barakos, "Numerical investigation of the aerodynamic interaction between a tiltrotor and a tandem rotor during shipboard operations," Aerospace Science and Technology, vol. 87, pp. 62-72, 2019.

[36] Y. Lu, T. Su, R. Chen, P. Li, and Y. Wang, "A method for optimizing the aerodynamic layout of a helicopter that reduces the effects of aerodynamic interaction," Aerospace Science and Technology, vol. 88, pp. 73-83, 2019.

[37] F. Farrasate, "Derivation of formulations 1 and $1 \mathrm{~A}$ of Farassat," NASA/TM Report no. TM-2007-214853, NASA, Washington, DC, USA, 2007.

[38] J. E. F. Williams and D. L. Hawkings, "Sound generation by turbulence and surfaces in arbitrary motion," Philosophical Transactions of the Royal Society A: Mathematical, Physical and Engineering Sciences, vol. 264, no. 1151, pp. 321-342, 1969.

[39] J. G. Leishman and T. S. Beddoes, “A generalized method for unsteady airfoil behavior and dynamic stall using the indicial method," in Proceedings of the 42th American Helicopter Society Annual Forum, Washington, DC, USA, June 1986.

[40] N. Hariharan and J. G. Leishman, "Unsteady aerodynamics of a flapped airfoil in subsonic flow by indicial concepts," in Proceedings of the 36th AIAA/ASME/ASCE/AHS/ASC Structures, Structural Dynamics, and Materials Conference, New Orleans, LA, USA, April 1995.

[41] I. E. Garrick, Propulsion of a Flapping and Oscillating Airfoil, Vol. 567, National Advisory Committee for Aeronautics, Langley Field, VA, USA, 1936.

[42] M. J. Bhagwat, R. A. Ormistion, H. A. Saberi, and H. Xin, "Application of CFD/CSD coupling for analysis of rotorcraft airloads and blade loads in maneuvering flight," in Proceedings of the 63th American Helicopter Society Annual Forum, Virginia Beach, VA, USA, May 2007. 


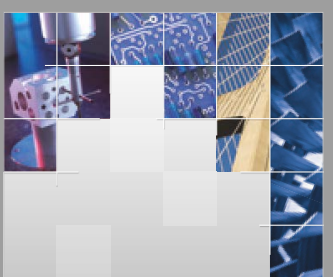

\section{Enfincering}


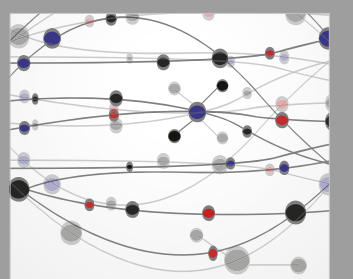

\section{Rotating \\ Machinery}

The Scientific World Journal

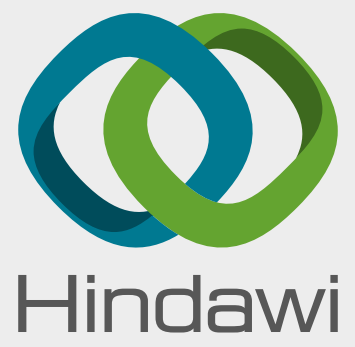

Submit your manuscripts at

www.hindawi.com
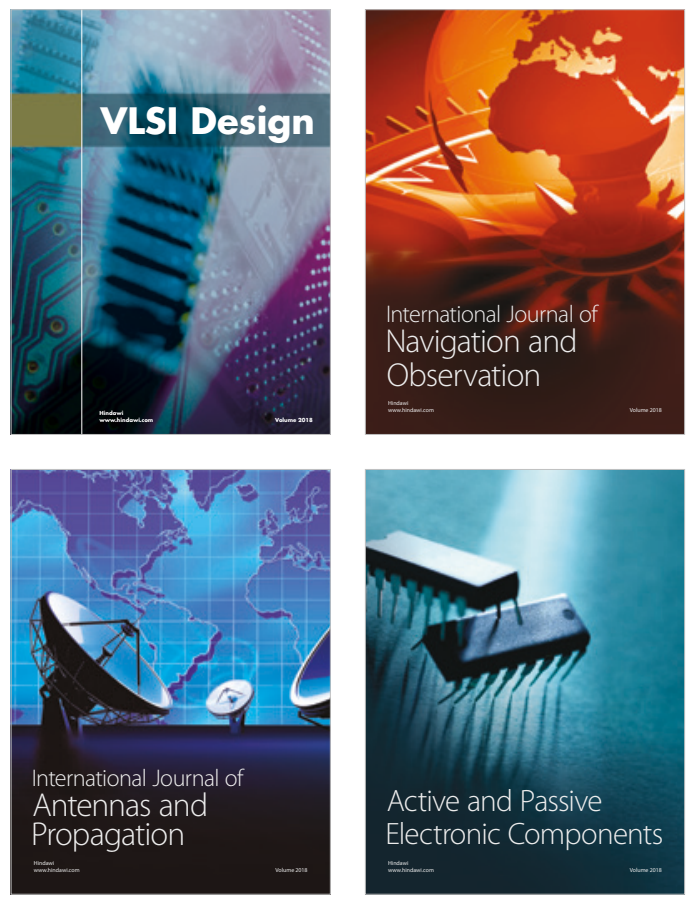
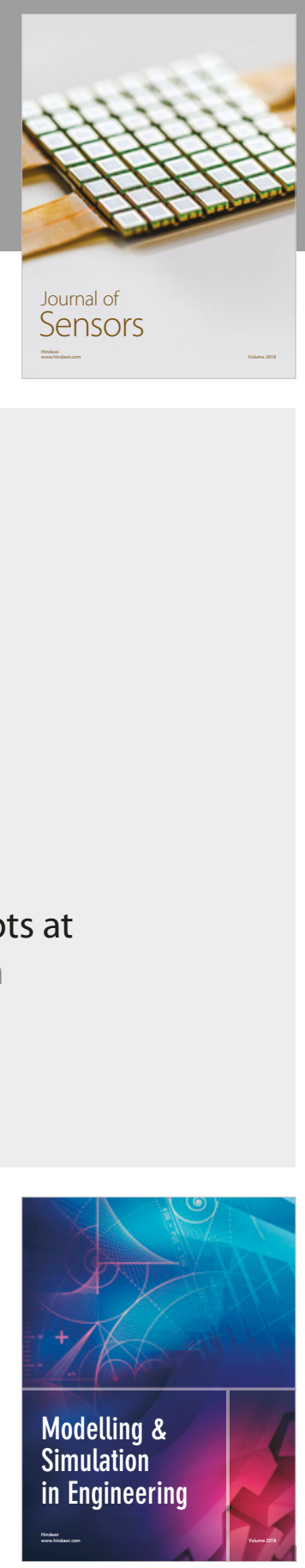

\section{Advances \\ Multimedia}
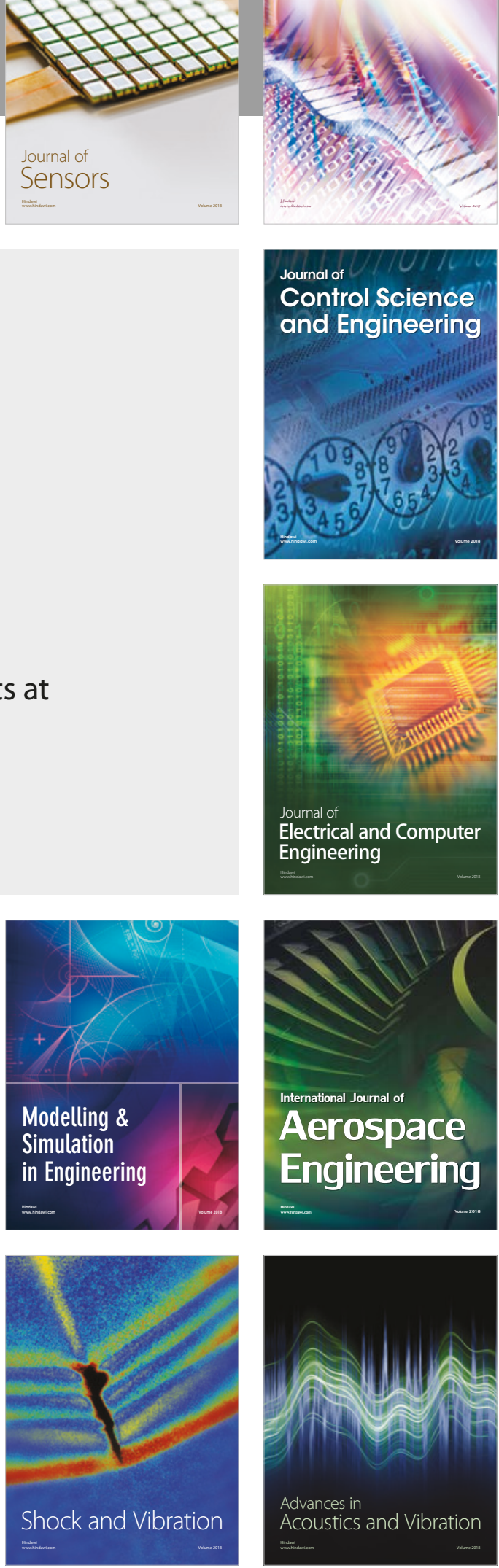